VOL. 50 (1994) [459-464]

\title{
GROUPS COVERED BY FINITELY MANY NILPOTENT SUBGROUPS
}

\author{
GÉRARD ENDIMIONI
}

Let $G$ be a finitely generated soluble group. Lennox and Wiegold have proved that $G$ has a finite covering by nilpotent subgroups if and only if any infinite set of elements of $G$ contains a pair $\{x, y\}$ such that $\langle x, y\rangle$ is nilpotent. The main theorem of this paper is an improvement of the previous result: we show that $G$ has a finite covering by nilpotent subgroups if and only if any infinite set of elements of $G$ contains a pair $\{x ; y\}$ such that $[x, n y]=1$ for some integer $n=n(x, y) \geqslant 0$.

\section{INTRODUCTION AND RESULTS}

Let $x$ and $y$ be elements of a group $G$ and let $n$ be a non-negative integer. As usual, $\left[x,_{n} y\right]$ is defined inductively by $[x, 0 y]=x$ and $\left[x,_{n+1} y\right]=[[x, n y], y]$, where $[x, y]=x^{-1} y^{-1} x y$. We say that $G$ is covered by a family of subgroups $\left(H_{i}\right)_{i \in I}$ if $G=\bigcup_{i \in I} H_{i}$. The family $\left(H_{i}\right)_{i \in I}$ is called a covering of $G$. The following characterisation for finitely generated soluble groups covered by finitely many nilpotent subgroups was obtained by Lennox and Wiegold [4]:

THEOREM A. Let $G$ be a finitely generated soluble group. Then the following properties are equivalent:

(i) $G$ is finite-by-nilpotent (that is, $G$ has a finite covering by nilpotent subgroups, by Lemma 5 below).

(ii) Any infinite set of elements of $G$ contains a pair $\{x, y\}$ which generate a nilpotent subgroup.

The main purpose of this note is to improve the previous result. We shall prove:

THEOREM 1.' Let $G$ be a finitely generated soluble group. Then the following properties are equivalent:

(i) $G$ has a finite covering by nilpotent subgroups.

(ii) Any infinite set of elements of $G$ contains a pair $\{x, y\}$ such that $[x, n y]=$ 1 for some integer $n=n(x, y) \geqslant 0$.

Received 15 February 1994

Copyright Clearance Centre, Inc. Serial-fee code: 0004-9729/94 $\$ A 2.00+0.00$. 
Note that this theorem is not true for an arbitrary group: the standard wreath product of a group of prime order $\boldsymbol{p}$ and an infinite elementary abelian $p$-group satisfies (ii) (this group is locally nilpotent) but does not satisfy (i) by Lemma 5 below (the centre is trivial).

The origin of the previous results is a problem of $\boldsymbol{P}$. Erdös [6]. Associate with a group $G$ a graph $\Gamma(G)$ in this way: the vertices of $\Gamma(G)$ are the elements of $G$, and two vertices $x, y$ are connected by an edge if and only if $[x, y] \neq 1$.

Suppose that $\Gamma(G)$ contains no infinite complete subgraph (that is, any infinite set of elements of $G$ contains a pair $\{x, y\}$ such that $[x, y]=1$ ); is there then a finite bound on the cardinality of complete subgraphs of $\Gamma(G)$ ?

Neumann [6] solved the problem in the affirmative by proving that if $\Gamma(G)$ contains no infinite complete subgraph, then $G$ has a finite covering by abelian subgroups. Therefore, if $G$ is covered by $n$ abelian subgroups, the order of a complete subgraph of $\Gamma(G)$ is at most $n$. Now consider the graph $\Gamma^{*}(G)$, where the vertices are the elements of $G$, and two vertices $x, y$ are connected by an edge if and only if $[x, n y] \neq 1$ and $[y, n] \neq 1$ for every integer $n \geqslant 0$. By observing that $\Gamma^{*}(G)$ contains no infinite complete subgraph if and only if $G$ satisfies the property (ii) of Theorem 1 , we obtain at once the following consequence of the Theorem 1:

Corollary. Let $G$ be a finitely generated soluble group. Suppose that the graph $\Gamma^{*}(G)$ defined above contains no infinite complete subgraph. Then, there exists a finite bound on the cardinality of complete subgraphs of $\Gamma^{*}(G)$.

Now, consider an infinite group $G$. As was observed in [5], if for every pair $\{X, Y\}$ of infinite subsets of $G$ there exists $x \in X, y \in Y$ such that $[x, y]=1$, then $G$ is abelian. For finitely generated soluble groups, this result was extended in this way:

THEOREM B. [9] Let $k>0$ be an integer. Let $G$ be an infinite finitely generated soluble group such that, whenever $X, Y$ are infinite subsets of $G$, there exist $x \in X$, $y \in Y$ such that $\left[x,{ }_{k} y\right]=1$. Then $G$ is a $k$-Engel group (that is, $\left[x,{ }_{k} y\right]=1$ for all $x, y$ in $G$ )

By a result of Gruenberg [2], it is well-known that every finitely generated soluble Engel group is nilpotent. Therefore, under the assumptions of Theorem $B$, the group $G$ is nilpotent. As a consequence of Theorem 1 , we shall prove a result of a similar nature:

THEOREM 2. Let $G$ be an infinite finitely generated soluble group such that, whenever $X, Y$ are infinite subsets of $G$, there exist $x \in X, y \in Y$ and an integer $n \geqslant 0$ such that $[x, n y]=1$. Then $G$ is nilpotent. 


\section{SOME PRELIMINARY LEMMAS}

Let $u$ be an element of a group $G$. An element $x$ of $G$ is called a right Engel element with respect to $u$ if there exists an integer $n \geqslant 0$ such that $[x, n]=1$. Let $R_{u}(G)$ denote the set of all such elements. An element of $R(G):=\bigcap_{u \in G} R_{u}(G)$ is called a right Engel element. If the derived subgroup $G^{\prime}$ is nilpotent (in particular if $G$ is metabelian), then $R_{u}(G)$ is a subgroup of $G[7]$.

Lemma 1. Let $u, u_{1}, \ldots, u_{k}$ be arbitrary elements of a metabelian group $G$. Then

(i) $R_{u^{-1}}(G)=R_{u}(G)$.

(ii) $\bigcap_{t \in G} t^{-1}\left\{R_{u_{1}}(G) \cap \ldots \cap R_{u_{k}}(G)\right\} t \subseteq \bigcap_{t \in G} t^{-1} R_{u_{1} \ldots u_{k}}(G) t$.

(iii) If $G=\left\langle w_{1}, \ldots, w_{q}\right\rangle$ is finitely generated, we have

$$
R(G)=\bigcap_{t \in G} t^{-1}\left\{R_{w_{1}}(G) \cap \ldots \cap R_{w_{q}}(G)\right\} t .
$$

Proof: (i) It suffices to show the relation

$$
\left[x,{ }_{n} u^{-1}\right]=u^{n}\left[x,{ }_{n} u\right]^{(-1)^{n}} u^{-n}
$$

for arbitrary $u, x \in G$ and $n \geqslant 0$. Observe that our relation is true for $n \in\{0,1\}$ and suppose that $\left[x_{n-1} u^{-1}\right]=u^{n-1}[x, n-1 u]^{(-1)^{n-1}} u^{-n+1}$ for an integer $n>1$. Then

$$
\begin{aligned}
{\left[x_{n, n} u^{-1}\right] } & =\left[\left[x_{n-1} u^{-1}\right], u^{-1}\right]=\left[u^{n-1}\left[x_{, n-1} u\right]^{(-1)^{n-1}} u^{-n+1}, u^{-1}\right] \\
& =u^{n-1}\left[\left[x_{n-1} u\right]^{(-1)^{n-1}}, u^{-1}\right] u^{-n+1} .
\end{aligned}
$$

Since $[x, n-1 u]$ commutes with its conjugates, we can write

$$
\left[x, n u^{-1}\right]=u^{n-1}\left[[x, n-1 u], u^{-1}\right]^{(-1)^{n-1}} u^{-n+1} .
$$

But $\left[\left[x_{n-1} u\right], u^{-1}\right]=u\left[\left[x_{n-1} u\right], u\right]^{-1} u^{-1}$, hence we obtain

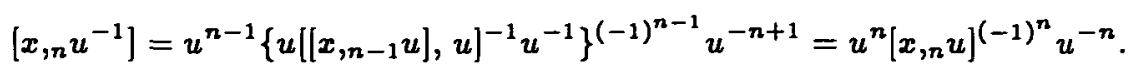

(ii) We show the assertion in the case $k=2$ : the assertion in the general case will follow at once by an easy induction on $k$. For convenience denote $u_{1}$ by $u$ and $u_{2}$ by $v$. Let $x$ be an element of $\bigcap_{t \in G} t^{-1}\left\{R_{u}(G) \cap R_{v}(G)\right\} t$. Since $\bigcap_{t \in G} t^{-1}\left\{R_{u}(G) \cap R_{v}(G)\right\} t$ is a normal subgroup of $G$, it suffices to prove that $x$ belongs to $R_{u v}(G)$. First note that $[x, u v]$ is an element of $\bigcap_{t \in G} t^{-1}\left\{R_{u}(G) \cap R_{v}(G)\right\} t$. Thus there exists an integer $n>0$ 
such that $\left[x, u v,_{n} u\right]=\left[x, u v,_{n} v\right]=1$. From the relations $[y, u v]=[y, u][y, v][y, u, v]$ and $[y, u, v]=[y, v, u]\left(y \in G^{\prime}\right)$, we deduce that $[x, 2 n u v]$ is a product of commutators of the form $\left[x, u v, r u^{\prime},{ }_{s} v^{\prime}\right]$, where $r+s \geqslant 2 n-1, r \geqslant s$ and $\left\{u^{\prime}, v^{\prime}\right\}=\{u, v\}$. But the previous inequalities imply $r \geqslant n$, hence $[x, 2 n u v]=1$ and so $x \in R_{u v}(G)$ as required.

(iii) Clearly, we have the inclusion $R(G) \subseteq \bigcap_{t \in G} t^{-1}\left\{R_{w_{1}}(G) \cap \ldots \cap R_{w_{q}}(G)\right\} t$. Conversely, to prove the inclusion $\bigcap_{t \in G} t^{-1}\left\{R_{w_{1}}(G) \cap \ldots \cap R_{w_{q}}(G)\right\} t \subseteq R(G)$, it must be shown that $\bigcap_{t \in G} t^{-1}\left\{R_{w 1}(G) \cap \ldots \cap R_{w_{q}}(G)\right\} t \subseteq R_{u}(G)$ for an arbitrary element $u \in G$. Write $u$ in the form of a product of elements in $\left\{w_{1}, \ldots, w_{q}\right\} \cup\left\{w_{1}^{-1}, \ldots, w_{q}^{-1}\right\}$ and apply (i) (ii): it follows that

$$
\bigcap_{t \in G} t^{-1}\left\{R_{w_{1}}(G) \cap \ldots \cap R_{w_{q}}(G)\right\} t \subseteq \bigcap_{t \in G} t^{-1} R_{u}(G) t
$$

Hence $\bigcap_{t \in G} t^{-1}\left\{R_{w_{1}}(G) \cap \ldots \cap R_{w_{q}}(G)\right\} t \subseteq R_{u}(G)$, so (iii) is proved.

Lemma 2. Let $G$ be a metabelian group satisfying the property (ii) of Theorem 1. Then

(i) $R_{u}(G)$ has finite index in $G$ for every $u \in G$.

(ii) If $G$ is finitely generated, $R(G)$ has finite index in $G$.

ProOF: (i) Suppose there exists $u \in G$ such that $\left|G: R_{u}(G)\right|$ is infinite and choose a right transversal $T$ of $R_{u}(G)$ in $G$. If $x^{-1} u x=y^{-1} u y(x, y \in T)$, then $\left[x y^{-1}, u\right]=1$, hence $x=y$ since $x y^{-1} \in R_{u}(G)$. Therefore, the set of conjugates of $u$ by elements of $T$ is infinite. Hence there exist $x, y \in T(x \neq y)$ and $n>0$ such that $\left[x^{-1} u x, n y^{-1} u y\right]=1$. We have

$$
\begin{aligned}
1 & =\left[y x^{-1} u x y^{-1},_{n} u\right]=\left[u\left[u, x y^{-1}\right]_{n} u\right]=\left[\left[u, x y^{-1}\right]_{n} u\right] \\
& =\left[\left[x y^{-1}, u\right]^{-1},_{n} u\right]=\left[\left[x y^{-1}, u\right]_{n} u\right]^{-1}=\left[x y^{-1}{ }_{n+1} u\right]^{-1}
\end{aligned}
$$

and so $x y^{-1} \in R_{u}(G)$, a contradiction.

(ii) Suppose that $G=\left\langle w_{1}, \ldots, w_{q}\right\rangle$. By (i), every subgroup $R_{w_{1}}(G), \ldots, R_{w_{q}}(G)$ has finite index in $G$, hence also $R_{w_{1}}(G) \cap \ldots \cap R_{w_{q}}(G)$ and $\bigcap_{t \in G} t^{-1}\left\{R_{w_{1}}(G) \cap \ldots \cap\right.$ $\left.R_{w_{q}}(G)\right\} t$. Using Lemma 1 (iii), we obtain the required result.

The following result is due to Lennox [4]:

Lemma 3. Let $G$ be a finitely generated soluble group and $A$ an abelian normal subgroup such that $G / A$ is polycyclic and $\langle a, x\rangle$ is polycyclic whenever $a \in A, x \in G$. Then $G$ is polycyclic. 
Lemma 4. Let $G$ be a finitely generated soluble group satisfying the property (ii) of Theorem 1. Then $G$ is polycyclic.

Proof: Denote by $d$ the derived length of $G$. First we show the lemma in the case $d \leqslant 2$. If $d \leqslant 1$, the result is obvious. Suppose now that $d=2$. By Lemma 2 , $|G: R(G)|$ is finite; hence $R(G)$ is finitely generated. Moreover $R(G)$ is a soluble Engel group and hence $R(G)$ is nilpotent [2]. Therefore we can say that $G$ is polycyclic-bypolycyclic so $G$ is polycyclic. Finally, use induction on $d$ in the general case. If $d>0$, put $A=G^{(d-1)}$. It follows from the inductive hypothesis that $G / A$ is polycyclic. Clearly, the derived length of $\langle a, x\rangle$ is at most 2 whenever $a \in A, x \in G$, hence $\langle a, x\rangle$ is polycyclic. Lemma 3 permits us to conclude that $G$ is polycyclic.

Finally, we shall need the following characterisation of groups covered by finitely many nilpotent subgroups (see [10] for the equivalence of (i) and (ii) and [3] for the equivalence of (ii) and (iii)):

LEMma 5. For an arbitrary group $G$, the following properties are equivalent:

(i) $G$ has a finite covering by nilpotent subgroups.

(ii) For some integer $c \geqslant 0$, the term $\zeta_{c}(G)$ of the upper central series of $G$ has finite index in $G$.

(iii) $G$ is finite-by-nilpotent.

\section{ProOFs OF THE THEOREMS}

Proof of Theorem 1: We have only to show that (ii) implies (i) since the converse is clearly true. Use induction on the derived length $d$ of $G$, the case $d=0$ being trivial. For $d>0$, it follows from the inductive hypothesis and Lemma 5 that there exists an integer $c \geqslant 0$ such that $\left|G / G^{(d-1)}: \zeta_{c}\left(G / G^{(d-1)}\right)\right|<\infty$. But in a finitely generated soluble group, the hypercentre coincides with the set of right Engel elements [1]; hence $\left|G / G^{(d-1)}: R\left(G / G^{(d-1)}\right)\right|$ is finite. Let $e$ denote the exponent of the quotient group $\left(G / G^{(d-1)}\right) / R\left(G / G^{(d-1)}\right)$. Therefore, for all $x, y \in G$, there exists an integer $m \geqslant 0$ such that $\left[x^{e},{ }_{m} y\right] \in G^{(d-1)}$. The subgroup $H=\left\langle\left[x^{e},{ }_{m} y\right], y\right\rangle$ is clearly metabelian. Hence $R(H)$ has finite index in $H$ by Lemma 2 . Denote by $f$ the exponent of $H / R(G)$. Thus there exists an integer $n \geqslant 0$ such that $\left[\left[x^{e},{ }_{m} y\right]^{f}{ }_{n} y\right]=1$. Since $\left[x^{e}{ }_{m} y\right]$ commutes with its conjugates, we obtain

$$
\left[\left[x^{e}{ }_{m} y\right]^{f}{ }_{n} y\right]=\left[\left[x^{e}{ }_{m} y\right],_{n} y\right]^{f}=1 .
$$

In other words, $\left[x^{e}, m_{m+n} y\right]$ belongs to the torsion group $\tau\left(G^{(d-1)}\right)$ of $G^{(d-1)}$. This means that the quotient group $\left\{G / \tau\left(G^{(d-1)}\right)\right\} / R\left(G / \tau\left(G^{(d-1)}\right)\right)$ has exponent dividing $e$ and so is finite. But $R\left(G / \tau\left(G^{(d-1)}\right)\right)$ coincides with the hypercentre of $G / \tau\left(G^{(d-1)}\right)$ by the result quoted above. Moreover, $G / \tau\left(G^{(d-1)}\right)$ satisfies the maximal condition 
on subgroups by Lemma 4 . Therefore we have $R\left(G / \tau\left(G^{(d-1)}\right)\right)=\zeta_{c^{\prime}}\left(G / \tau\left(G^{(d-1)}\right)\right)$ for some integer $c^{\prime} \geqslant 0$ and $\left|G / \tau\left(G^{(d-1)}\right): \zeta_{c^{\prime}}\left(G / \tau\left(G^{(d-1)}\right)\right)\right|$ is finite. We deduce from Lemma 5 that $G / \tau\left(G^{(d-1)}\right)$ is finite-by-nilpotent. But $G$ satisfies the maximal condition (Lemma 4) hence $\tau\left(G^{(d-1)}\right)$ is finite and so $G$ is finite-by-nilpotent. Finally, Lemma 5 shows that $G$ has a finite covering by nilpotent subgroups, as required.

Proof OF TheOREM 2: It suffices to show that $\zeta^{*}(G)=G$, where $\zeta^{*}(G)$ is the hypercentre of $G$. Clearly, $G$ satisfies the property (ii) of Theorem 1 , hence $G$ has a finite covering by nilpotent subgroups. It follows from Lemma 5 that $\zeta^{*}(G)$ has finite index in $G$. In particular, $\zeta^{*}(G)$ is infinite. Let $x, y$ be elements of $G$. Subsets $x \zeta^{*}(G)$ and $y \zeta^{*}(G)$ are infinite, hence there exist $u, v \in \zeta^{*}(G), n \geqslant 0$, such that $\left[x u,{ }_{n} y v\right]=1$. This implies $[x, n y] \in \zeta^{*}(G)$, so $G / \zeta^{*}(G)$ is an Engel group. But it is well-known that finite Engel groups are nilpotent (for example $[8,7.21]$ ), so $G / \zeta^{*}(G)$ is nilpotent. Since the centre of $G / \zeta^{*}(G)$ is trivial, we obtain $\zeta^{*}(G)=G$.

\section{REFERENCES}

[1] C.J.B. Brookes, 'Engel elements of soluble groups', Bull. London Math. Soc. 18 (1986), 7-10.

[2] K.W. Gruenberg, 'Two theorems on Engel groups', Proc. Cambridge Philos. Soc. 49 (1953), 377-380.

[3] P. Hall, 'Finite-by-nilpotent groups', Proc. Cambridge Philos. Soc. 52 (1956), 611-616.

[4] J.C. Lennox and J. Wiegold, 'Extensions of a problem of Paul Erdös on groups', J. Austral. Math. Soc. Ser. A 31 (1981), 459-463.

[5] P. Longobardi, M. Maj and A.H. Rhemtulla, 'Infinite groups in a given variety and Ramsey's theorem', Comm. Algebra 20 (1992), 127-139.

[6] B.H. Neumann, 'A problem of Paul Erdös on groups', J. Austral. Math. Soc. Ser. A 21 (1976), 467-472.

[7] T.A. Peng, 'On groups with nilpotent derived groups', Arch. Math. 20 (1969), 251-253.

[8] D.J.S. Robinson, Finiteness conditions and generalized soluble groups (Springer-Verlag, Berlin, Heidelberg, New York, 1972).

[9] L.S. Spiezia, 'Infinite locally soluble k-Engel groups', Atti Accad. Naz. Lincei Rend. Cl. Sci. Fis. Mat. Natur. (9) Mat. Appl. 3 (1992), 177-183.

[10] M.J. Tomkinson, 'Hypercentre-by-finite groups', Publ. Math. Debrecen 40 (1992), 313-321.

Université de Provence

UFR-MIM URA-CNRS 225

3 place Victor Hugo

F-13331 Marseille Cedex 3

France 